\title{
Composite Grip Strength as a Marker of Outcome in Patients Surgically Treated for Degenerative Cervical Myelopathy
}

\author{
Arun John Paul ${ }^{1}$, Rohit Amritanand ${ }^{1}$, Prabakaran Margabandhu ${ }^{2}$, \\ Reka Karuppusami ${ }^{3}$, Kenny Samuel David ${ }^{1}$, Venkatesh Krishnan ${ }^{1}$ \\ ${ }^{1}$ Spinal Disorders Surgery Unit, Department of Orthopaedics, Christian Medical College, Vellore, India \\ ${ }^{2}$ Department of Hand Physiotherapy, Christian Medical College, Vellore, India \\ ${ }^{3}$ Department of Biostatistics, Christian Medical College, Vellore, India
}

Study Design: Retrospective case series.

Purpose: This study aimed to examine the efficacy of composite grip strength as a marker of surgical outcome in patients with moderate to severe degenerative cervical myelopathy.

Overview of Literature: Degenerative cervical myelopathy causes loss of dexterity, muscle strength, and sensations in the hand. The impact of surgical management on improvement in composite grip strength has received scant attention.

Methods: This retrospective study was performed on degenerative cervical myelopathy patients with a complete composite grip strength assessment between January 2013 to January 2019. The Biometrics E-link hand kit was used for the assessment. The following parameters were measured: maximum grip strength, sustained grip strength, three-jaw pinch, maximum key pinch, and sustained key pinch. The pre- and postoperative functional status was assessed using the Nurick grade and the modified Japanese Orthopaedic Association (mJOA) score.

Results: A total of 40 patients were included in the study. The mean patient age was 51.9 years. The mean preoperative Nurick grade was 3.5 and the mJOA score was 10.9. The anterior approach was used in 25 patients, and the posterior approach was used in 15 patients. Four patients developed complications. Degenerative cervical myelopathy resulted in decreased handgrip and pinch strength as compared to normative Indian data. There was a significant improvement in the postoperative composite grip strength for all five parameters. There was no differential improvement between the anterior and posterior surgical groups. The improvement in the composite grip strength correlated with the improvement in functional scores.

Conclusions: Composite grip strength analysis is an objective method for assessing the impact of degenerative cervical myelopathy on grip strength and monitoring the postoperative improvement. Decompressive surgery resulted in global improvement in all the parameters of composite grip strength.

Keywords: Spinal cord diseases; Surgical decompression; Grip strength; Pinch strength

Received May 20, 2020; Revised Jun 28, 2020; Accepted Jun 28, 2020

Corresponding author: Rohit Amritanand

Spinal Disorder Surgery Unit, Christian Medical College, Vellore, Tamil Nadu-632004, India

Tel: +91-416-2282731, Fax: +91-9500993722, E-mail: rohit@cmcvellore.ac.in 


\section{Introduction}

Degenerative cervical myelopathy (DCM) is the most common cause of non-traumatic neurological dysfunction of the spinal cord in adults [1]. The degenerative changes that occur in this condition involve the intervertebral discs, vertebral bodies, facet joints, hypertrophy of the ligamentum flavum, and ossification of the posterior longitudinal ligament (OPLL) [2]. The ensuing myelopathy, in addition to balance and gait disturbances, may lead to the inability to use hands effectively for daily activities. This is attributable to a combined loss of dexterity, muscle strength, and sensations in the hand, the so called "myelopathy hand" first described by Ono et al. [3]. Decompressive surgery is offered as the treatment option in DCM. Given the magnitude of the surgery, it is important to establish the disease severity, the impact of surgical intervention, and the expected treatment outcomes [4]. To document this, several tests of hand function have been described. These tests determine manual dexterity $[5,6]$, the ability to perform rapid grasp and release $[7,8]$, motor conduction measurements [9], and proprioceptive function of the hand [10]. Surprisingly, the impact of DCM on grip strength has received limited attention. Recently, Yoo et al. [11] reported grip strength in patients undergoing anterior cervical discectomy and fusion. However, they included patients with both radiculopathy and myelopathy. Egwu et al. [12] have described the impact of cervical spondylosis on grip strength in patients who presented to their physiotherapy department; however, no surgical treatment was given to further evaluate its impact. The impact of surgical management of DCM on grip strength has been described by Liu et al. [13]; however, only cursorily and as part of a battery of other tests while comparing the efficacy of two different surgical decompressive strategies on DCM. Our study focuses on the outcomes of surgery on grip strength in patients with moderate to severe myelopathy (modified Japanese Orthopaedic Association $[\mathrm{mJOA}] \leq 14)[14]$ because we believe that the combined impact of DCM on hand function is most profound in these patients. We used detailed and extensive computerbased assessment of composite grip strength (CoGS) that included grip and pinch strength assessment for the same.

Therefore, this study aimed to examine the efficacy of CoGS as a marker of DCM surgery outcome. The objectives were to study the impact of DCM on CoGS; to assess the impact of surgery on improvement of CoGS in patients with DCM; to compare the impact of age, sex, and surgical approach on CoGS; and analyze the correlation of CoGS improvement with the improvement in functional scores.

\section{Materials and Methods}

This retrospective study was conducted with approval from the Institutional Review Board at the Spinal Disorders Surgery Unit, Department of Orthopaedics, Christian Medical College, Vellore, India (IRB approval no., 12048). Informed consent was obtained from all the participants included in the study. The CoGS assessment was performed by a senior trained hand physiotherapist. An E-Link evaluation and exercise system, V900S supplied by Biometrics Limited, Gwent, United Kingdom was used for the CoGS assessment. It is a comprehensive computerized evaluation system with electronic instrumentation for isometric pinch and grip strength measurements. It connects to a standard computer and is easy to set up and use. The E-Link Hand Kit (H400) that is the automated evaluation part of the system that includes a Pinchmeter (P100) and Dynamometer (G100) was used. The modalities measured were maximum grip strength (GSmax), sustained grip strength (GSsust), three-jaw pinch (3JP), maximum key pinch (KPmax), and sustained key pinch (KPsust) (Figs. 1, 2). They were all measured in kilograms. The CoGS assessment procedure was first explained and then demonstrated to all the subjects. The subjects were seated in chairs, the forearm rested on the table with elbow at $90^{\circ}$ flexion, and forearm in the mid-prone position. They were asked to squeeze the handle of the dynamometer as hard as they could (GSmax). The dynamometer readings at the second position were considered for the analysis. GSsust was the force exerted over the final 3 seconds when the patient performed 5 -second grip strength test. The pinch strength was measured with the two following types of pinches: 3JP and key pinch; 3JP is measured with the index and the middle fingers on one side and the thumb on the other side; and key pinch is measured with the thumb on one side and the lateral side of the index finger on the other side with the patient making a fist. KPsust is the average force exerted over the final 3 seconds when the patient performs a 5-second key pinch. Each test was performed thrice, and the mean value was considered as the final value for data analysis. The time taken for the complete CoGS assessment in both hands was about 15-20 minutes. 
(A)

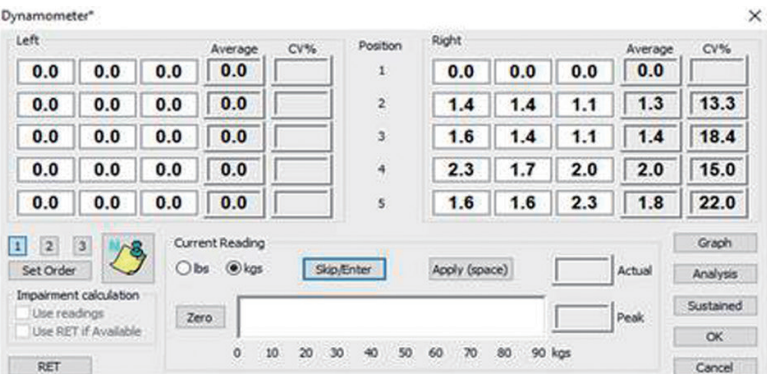

Pinchmeter:

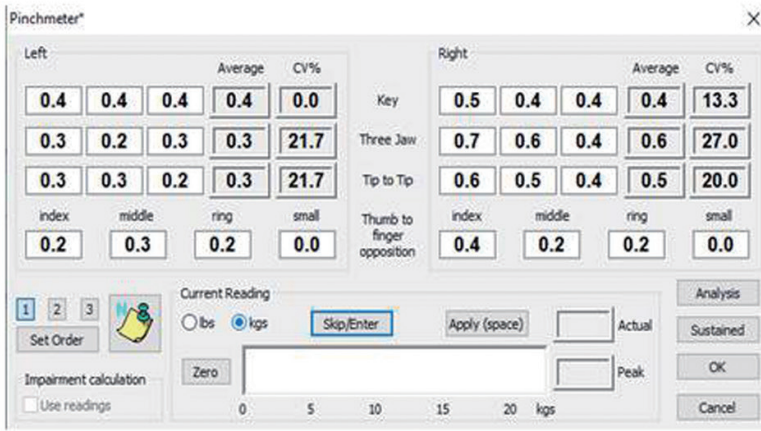

(B)
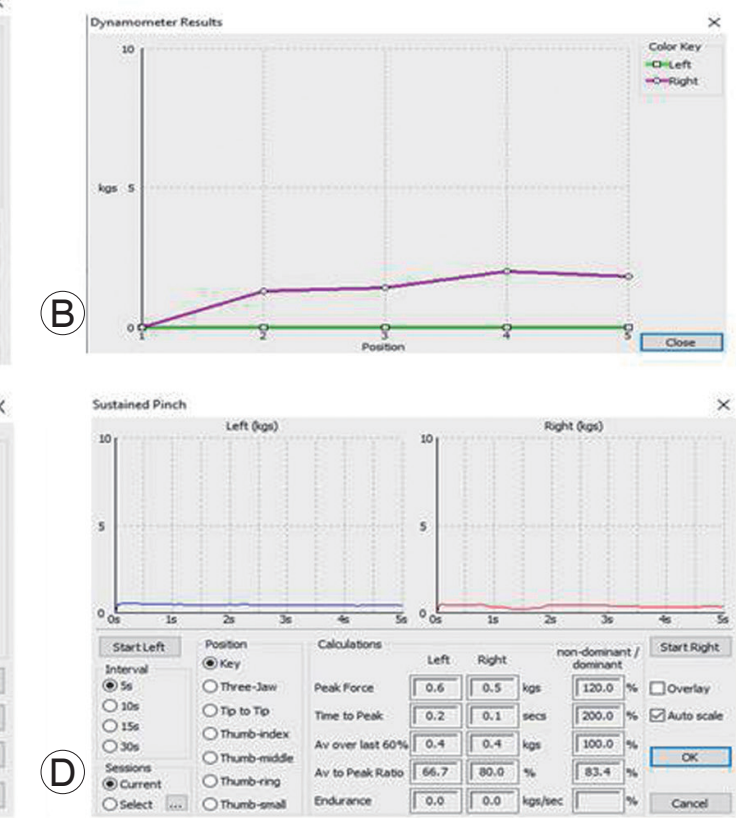

Fig. 1. (A-D) Preoperative composite grip strength assessment of a patient with degenerative cervical myelopathy.

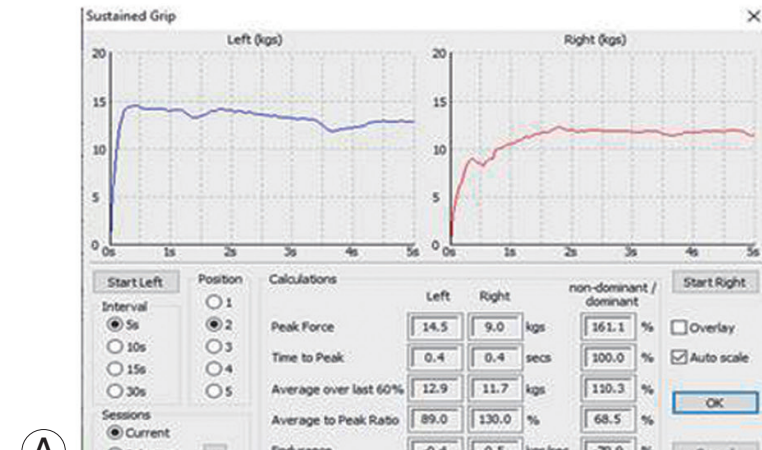

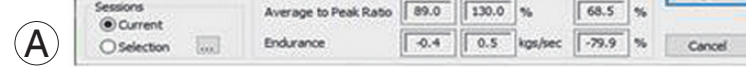

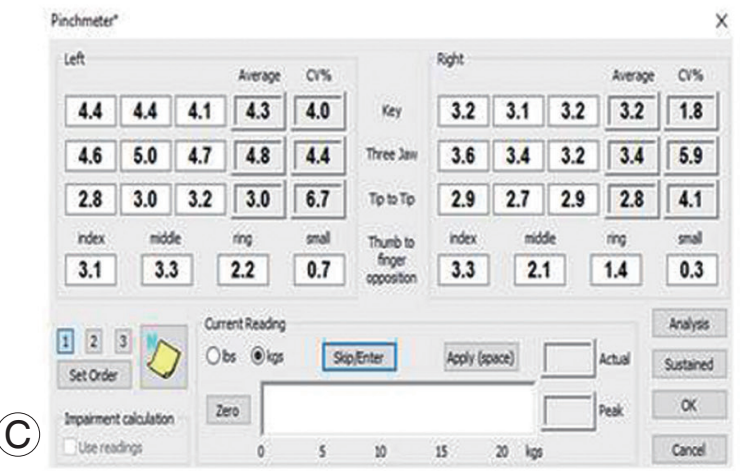

(B)
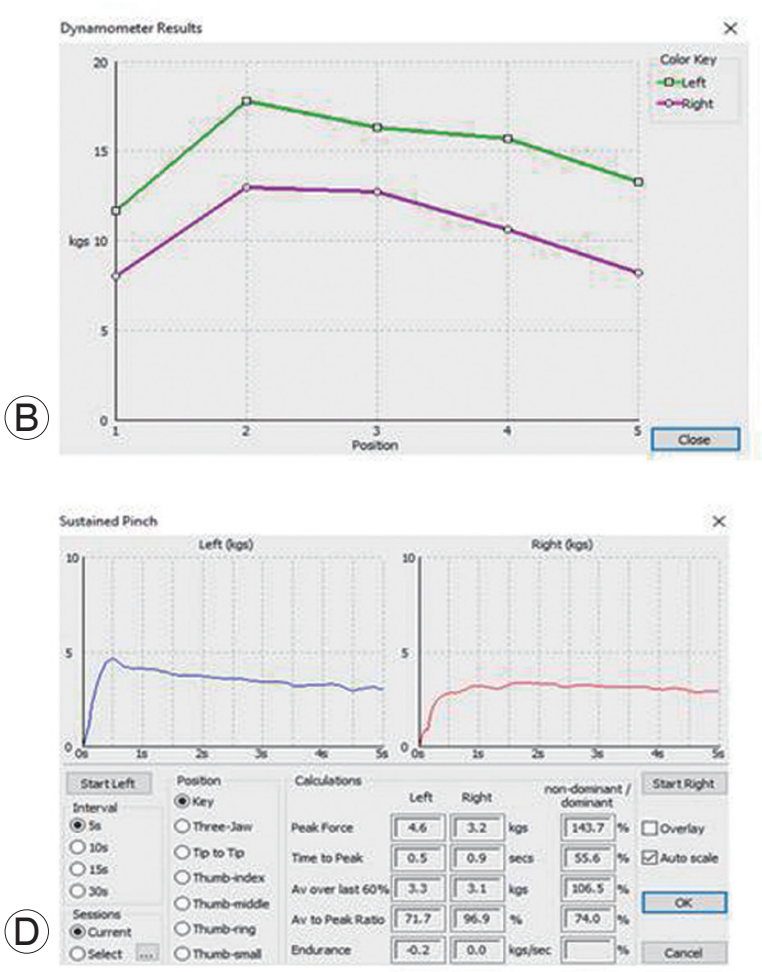

Fig. 2. (A-D) Postoperative composite grip strength assessment of a patient with degenerative cervical myelopathy.

As per the inclusion criteria, patients with clinicoradiological diagnosis of moderate to severe DCM (mJOA $\leq 14)$, those with a complete CoGS analysis preoperatively and postoperatively at a minimum of 6 months after surgery were enrolled. As per the exclusion criteria, those with hand symptoms caused by other coexisting conditions, such as a cerebrovascular accident and carpal tunnel syndrome; those with incomplete CoGS assessment; and those with less than 6-month follow-up after surgery were excluded. From January 2013 to January 2019, a total 
of 111 patients with DCM were operated at our center. Of these, the postoperative grip strength analysis results were unavailable for 46 patients, mild myelopathy (mJOA 1517) was present in 17 , postoperative grip strength analysis was performed $<6$ months after the surgery in seven, and there was a co-existing cerebrovascular accident in one. Forty patients who fulfilled the eligibility criteria were included in the study.

With respect to statistical analyses, mean ( \pm standard deviation) values were calculated for continuous data and median (interquartile range) were estimated for non-normally distributed data. Numbers (percentages) were reported for the categorical data. The change from preoperative to postoperative data for the following parameters (GSmax, GSsust, 3JP, KPmax, and KPsust) was assessed using non-parametric Wilcoxon signed-rank test. Parameters were compared between the anterior and posterior approach in the preoperative and postoperative groups. Moreover, the two groups were compared using the Mann-Whitney test. The change between the preoperative and postoperative functional scores for Nurick grade, mJOA, and upper limb motor score was assessed using paired $t$-test. The Pearson correlation coefficient $(r)$ was used to describe the relationship between CoGS parameters and functional outcomes (Nurick grade, mJOA score). Data entry was performed in the Epidata ver. 3.1 (The EpiData Association, Odense, Denmark). All analyses were performed using IBM SPSS software ver. 21.0 (IBM Corp., Armonk, NY, USA) and Stata ver. 13.0 (Stata Corp., College Station, TX, USA).

\section{Results}

A total of 40 patients were included in the study. Table 1 shows the preoperative clinical and functional details of the patients. The mean patient age was 51.9 years (range, 20-64 years), and the study population included 33 men and seven women. All the patients were right-hand dominant. During the initial analysis, we divided them into the following two groups based on their age: $\leq 55$ years $(n=20)$ and $>55$ years $(n=20)$. The analysis showed no significant differential improvement in the CoGS pre- and postoperatively between the two age groups. A similar analysis was performed to compare male and female subjects that showed no significant differential improvement. Moreover, the results of the right and left hands were comparable. Thus, for simplicity and clarity, we considered the
Table 1. Preoperative clinical and functional details

\begin{tabular}{|cc|}
\hline Characteristic & Value \\
\hline Age (yr) & $51.9 \pm 10.6$ \\
\hline Sex & \\
\hline \multicolumn{1}{|c|}{ Male } & $33(82.5)$ \\
\hline Female & $7(17.5)$ \\
\hline Follow-up (mo) & $24(6-72)$ \\
\hline Diagnosis & \\
\hline Cervical spondylotic myelopathy & $28(70.0)$ \\
\hline Intervertebral disc prolapse & $7(17.5)$ \\
\hline Ossified posterior longitudinal ligament & $5(12.5)$ \\
\hline Hand symptoms & \\
\hline Weakness & $30(75.0)$ \\
\hline Numbness & $24(60.0)$ \\
\hline Clumsiness & $31(77.5)$ \\
\hline No hand symptoms & $4(10.0)$ \\
\hline Upper limb motor score & $3.5 \pm 0.7$ \\
\hline
\end{tabular}

Values are presented as mean \pm standard deviation, number (\%), or mean (range).

Table 2. CoGS parameters comparison between subjects preoperatively and at final follow-up postoperatively

\begin{tabular}{lccc} 
CoGS parameters $(\mathrm{kg})$ & $\begin{array}{c}\text { Preoperative } \\
(\mathrm{n}=40)\end{array}$ & $\begin{array}{c}\text { Postoperative } \\
(\mathrm{n}=40)\end{array}$ & $p$-value \\
\hline Maximum grip strength & $15.5(8.6-27.3)$ & $24.2(18.7-30.8)$ & $<0.0001$ \\
\hline Sustained grip strength & $16.2(10.0-23.0)$ & $20.9(15.2-27.5)$ & $<0.0001$ \\
\hline Three-jaw pinch & $3.4(1.9-4.7)$ & $4.7(3.4-6.2)$ & $<0.0001$ \\
Maximum key pinch & $4(2.2-5.2)$ & $5.2(4.3-6.3)$ & $<0.0001$ \\
\hline Sustained key pinch & $3.1(1.8-4.9)$ & $4.3(3.6-5.4)$ & 0.001 \\
\hline
\end{tabular}

Values are presented as median (interquartile range). A $p$-value is obtained from non-parametric Wilcoxon signed-rank test.

CoGS, composite grip strength.

values of the right hand for all the patients, considering them as a single cohort. The mean follow-up duration was 24 months (range, 6-72 months). The most common diagnosis was cervical spondylotic myelopathy followed by intervertebral disc prolapse and OPLL. Ninety percent of the DCM patients had hand symptoms. The mean Nurick grade was 3.5. The mean JOA score was 10.9 (severe impairment). The mean upper limb motor component score of the mJOA score was 2.6 out of 5 . The grip and pinch strength of all the individuals were calculated preoperatively and at the final postoperative visit and compared 
Table 3. CoGS parameters comparison between subjects with anterior and posterior surgical approach

\begin{tabular}{|c|c|c|c|c|}
\hline CoGS parameters (kg) & Anterior approach $(\mathrm{n}=25)$ & Posterior approach ( $\mathrm{n}=15)$ & $p$-value ${ }^{\text {a) }}$ & $p$-value ${ }^{\text {b) }}$ \\
\hline Maximum grip strength & & & & 0.76 \\
\hline Preoperative & $23.0(13.6-28.4)$ & $14.0(1.3-15.2)$ & 0.009 & \\
\hline Postoperative & $26.2(22.2-34.5)$ & $17.9(13.0-23.2)$ & 0.004 & \\
\hline Sustained grip strength & & & & 0.98 \\
\hline Preoperative & $19.9(12.5-24.2)$ & $10.4(0.6-16.7)$ & 0.009 & \\
\hline Postoperative & $24.3(19.7-29.2)$ & $15.0(11.7-18.6)$ & 0.023 & \\
\hline Three-jaw pinch & & & & 0.31 \\
\hline Preoperative & $3.5(2.9-4.7)$ & $2.6(0.2-4.1)$ & 0.029 & \\
\hline Postoperative & $4.8(3.3-6.4)$ & $4.3(3.4-5.0)$ & 0.006 & \\
\hline Maximum key pinch & & & & 0.66 \\
\hline Preoperative & $4.4(3.5-5.6)$ & $1.9(0.4-4.5)$ & 0.007 & \\
\hline Postoperative & $5.3(4.6-7.5)$ & $4.6(3.5-5.4)$ & 0.017 & \\
\hline Sustained key pinch & & & & 0.32 \\
\hline Preoperative & $4.2(2.5-5.5)$ & $1.6(0.2-3.2)$ & 0.003 & \\
\hline Postoperative & $5.1(3.8-6.7)$ & $4.1(2.8-4.7)$ & 0.028 & \\
\hline
\end{tabular}

Values are presented as median (interquartile range).

CoGS, composite grip strength.

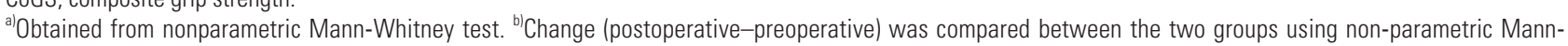
Whitney test.

Table 4. Preoperative and postoperative functional scores comparison between the subjects

\begin{tabular}{|c|c|c|c|}
\hline Functional scores & Preoperative $(n=40)$ & Postoperative $(n=40)$ & $p$-value \\
\hline Nurick grade & $3.5 \pm 0.7$ & $2.3 \pm 0.8$ & $<0.0001$ \\
\hline Modified Japanese Orthopaedic Association score & $10.9 \pm 2.7$ & $14.8 \pm 1.8$ & $<0.0001$ \\
\hline Upper limb motor score & $2.6 \pm 1.3$ & $4.2 \pm 0.9$ & $<0.0001$ \\
\hline
\end{tabular}

Values are presented as mean \pm standard deviation. A $p$-value is obtained from paired $t$-test.

(Table 2). Of the 40 patients, 25 underwent surgery via the anterior approach and 15 via the posterior approach. Their preoperative and postoperative grip and pinch strength analysis were performed separately and compared for any differences in the outcome (Table 3).

The preoperative and postoperative functional status was compared using Nurick grade and mJOA scores (Table 4). The mean preoperative Nurick grade was 3.5, and the mean postoperative Nurick grade was 2.3. The mean preoperative mJOA score was 10.9 , with the upper limb motor score being 2.6 out of 5 . The mean postoperative mJOA score was 14.8, with the upper limb motor score being 4.2 out of 5 . The correlation between the improvement in CoGS parameters and functional scores has been demonstrated using a scatter plot (Figs. 3, 4). It shows a positive correlation between the improvement in CoGS and improvement in functional scores (Nurick grade and mJOA score)

Table 5 shows the surgical details of all the patients. The anterior approach was preferred when $<3$ levels needed surgery, and the posterior approach was used when $>3$ levels needed surgery. Three-level surgeries were performed using either of the two approaches. Three cases showed complications. One patient developed C5-C6 palsy postoperatively and had recovered at the 6-month follow-up. The second patient sustained a dural tear for which he underwent surgery and thereafter recovered. The third patient developed a wound infection. He underwent wound debridement and implant removal following which his infection resolved. 
Table 5. Surgical details of the patients

\begin{tabular}{ll} 
Variable & \multicolumn{1}{c}{ Value } \\
Surgical approach & \\
\hline Anterior & $25(62.5)$ \\
\hline Age (yr) & $50.7(28-64)$ \\
\hline Male:female & $21: 4$ \\
\hline Posterior & $15(37.5)$ \\
\hline Age (yr) & $54(20-63)$ \\
\hline Male:female & $12: 3$ \\
\hline No. of levels operated [anterior/posterior] & \\
\hline 1 & $12(30)[12 / 0]$ \\
\hline 2 & $8(20)[8 / 0]$ \\
\hline 3 & $12(30)[5 / 7]$ \\
\hline 4 & $6(15)[0 / 6]$ \\
\hline 5 & $2(5)[0 / 2]$ \\
\hline Complications & $1(2.5)$ \\
\hline Dural tear & $1(2.5)$ \\
\hline Wound infection & $1(2.5)$ \\
\hline Neurological deficit & \\
\hline
\end{tabular}

Values are presented as number (\%) or mean (range), unless otherwise stated.

\section{Discussion}

DCM results in "myelopathy hand" that represents combined loss of dexterity, strength, and sensations in the hand [2]. This study demonstrates the impact of DCM and the outcome of decompressive surgery on grip strength. Our analysis showed decreased CoGS in all five parameters preoperatively. There was significant postoperative improvement in all the parameters. Comparison of the anterior and posterior surgical groups did not show any differential improvement in any of the five parameters. However, the posterior surgery patients had significantly low CoGS values in all five parameters preoperatively and postoperatively. This can be explained by the fact that all the patients in the posterior surgery group had multi-level involvement (three or more levels) and poorer functional scores as compared to those in the anterior surgery group. All the patients in the anterior group underwent 1-3 level surgeries, while the posterior group patients underwent 3-5 level surgeries. The mean mJOA score of the anterior group was 11.8 and that of the posterior group was 9.4 ( $p$ value $=0.004)$. Nevertheless, both the groups showed postoperative improvement in hand function. This showed that even though the anterior approach is preferred be- cause of the direct decompression it provides, the CoGS improvement was similar as that with the posterior approach. Assessment of the patients' functional status using the Nurick and mJOA scores showed significant postoperative improvement in all the patients. The overall mean Nurick grade improved from 3.5 (median=3) to 2.3 (median=2), and the mean mJOA score improved from 10.9 (median=11) that indicates the severe category to 14.8 (median=15) that indicates the mild category. The upper limb motor component of the mJOA score improved from 2.6 to 4.2 out of 5 . The CoGS improvement was positively correlated with an improvement in the functional scores. (Figs. 3, 4)

The normative data for Indian population for grip strength, 3JP, and key pinch in the age group of 18-30 years was presented by Mullerpatan et al. [15]. The GSmax, 3JP, and, KPmax were $33.6 \mathrm{~kg}$ (male) and $19.5 \mathrm{~kg}$ (female), $6.5 \mathrm{~kg}$ (male) and $4.6 \mathrm{~kg}$ (female), and $6.9 \mathrm{~kg}$ (male) and $4.8 \mathrm{~kg}$ (female), respectively. The differences between the values for male and female subjects were significant. The preoperative GSmax, 3JP, and KPmax in our study were $15.5 \mathrm{~kg}, 3.4 \mathrm{~kg}$, and $4 \mathrm{~kg}$, respectively, that is low compared to the normative data. However, the postoperative GSmax, 3JP, and KPmax in our study was comparable to the normative data $(24.2 \mathrm{~kg}, 4.7 \mathrm{~kg}$, and $5.2 \mathrm{~kg}$, respectively) considering that the mean patient age in our study was 51.9 years. There was no significant sex-based difference in our study, probably because our study group was relatively small with fewer female subjects (only seven out of 40).

The diagnostic and prognostic value of grip strength has been well established [16]. It is known to influence several parameters, including prediction of the length of hospital stay [17], all cause death [18], and cancer survivorship [19]. In fact, Bohannon [20] has described it as a "vital sign" for middle-aged and older individuals. Therefore, its assessment in virtually any disease condition is relevant. It also looks specifically at the grip and pinch strength that are specific for hand function as opposed to functional scores, such as the Nurick grade and mJOA that quantify the overall disease severity. Its value also stems from the fact that its assessment is simple, quick, and objective unlike the more cumbersome tests for assessing the hand function [21]. Furthermore, Tremayne et al. [22] have shown a strong association between grip strength and overall hand function, making it a surrogate marker or perhaps even a potential substitute for the more elaborate 

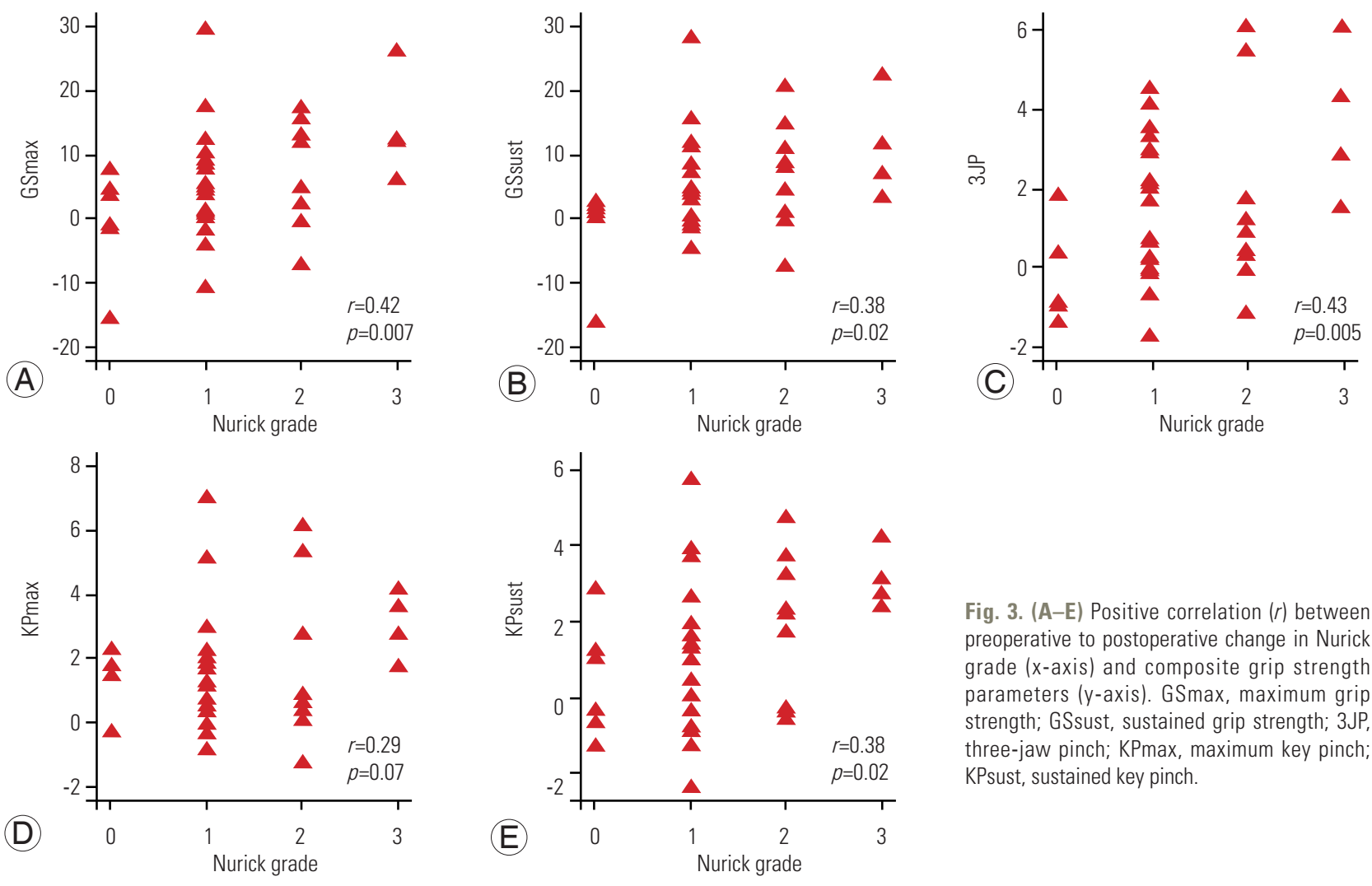

Fig. 3. (A-E) Positive correlation ( $r$ ) between preoperative to postoperative change in Nurick grade ( $\mathrm{x}$-axis) and composite grip strength parameters (y-axis). GSmax, maximum grip strength; GSsust, sustained grip strength; 3JP, three-jaw pinch; KPmax, maximum key pinch; KPsust, sustained key pinch.
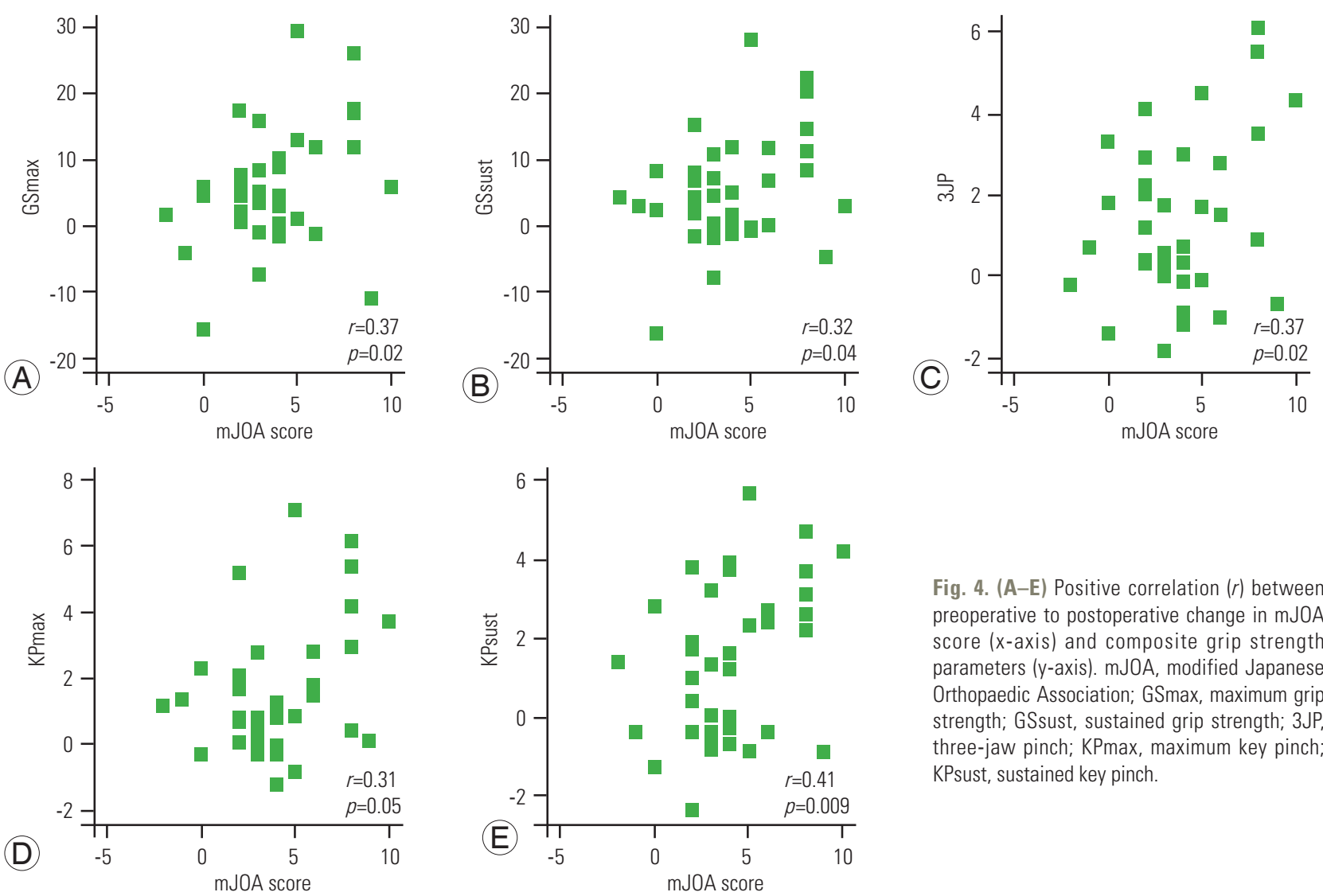

Fig. 4. (A-E) Positive correlation ( $r$ ) between preoperative to postoperative change in $\mathrm{mJOA}$ score ( $x$-axis) and composite grip strength parameters (y-axis). mJOA, modified Japanese Orthopaedic Association; GSmax, maximum grip strength; GSsust, sustained grip strength; 3JP, three-jaw pinch; KPmax, maximum key pinch; KPsust, sustained key pinch. 
and time-consuming tests [5], such as the grip and release test, nine-hole peg test, Jebsen-Taylor test, natural prehension movement, and 10-subtests assessment. The impact of surgery on DCM in improving the hand grip strength has received limited attention. We have attempted to study this by deploying the CoGS assessed by the E-Link system. This instrument has been used for assessing several other conditions that affect the hand function, such as rheumatoid arthritis [23], stroke [24], and brachial plexus injury [25]. It is a comprehensive computerized evaluation system. The hand kit is accurate and sensitive, registering forces as small as $0.05 \mathrm{~kg}$; therefore, patients may be objectively tested much earlier in the rehabilitation process. It is easy to set up, use, and comprehend. Moreover, it is easy to explain to the patients irrespective of their educational background and comprehension ability. It increases the speed and accuracy of data collection and reporting when compared to other tests of hand dexterity. The patients have a visual representation of the improvement in their hand function by looking at their preoperative and postoperative grip strength values. This motivates them by providing positive feedback and improving compliance.

This study has certain limitations. Owing to the retrospective nature of data collection and patient followup patterns, we had no control over the preoperative and postoperative CoGS assessment. It was not a part of the standard of care for DCM management; therefore, 46 of 111 patients (41\%) did not have complete CoGS assessment and could not be included in the study. Further, we did not have a control group that would have contributed more to the value of the study. Along with the abovementioned limitations, the present study has the following strengths: we exclusively investigated the impact of moderate to severe DCM and effect of surgery on CoGS. A standardized assessment tool was used for the entire duration of the study. The computerized assessment reduced subjectivity and improved speed and reliability of reporting.

\section{Conclusions}

CoGS analysis is an efficient and objective method for the assessment of hand function. It can be used as a tool to assess the severity of impact of DCM on hand function and as a baseline parameter to monitor the postoperative improvement along with other functional scores, such as the Nurick grade and the mJOA score. More research on serial monitoring of CoGS postoperatively and impact of formal occupational and physical therapy training on CoGS improvement is warranted to obtain further insights on this subject.

\section{Conflict of Interest}

No potential conflict of interest relevant to this article was reported.

\section{Author Contributions}

Conception and design: AJP, RA, PM, KSD, VK; data acquisition: AJP, RA, PM, KSD, VK; analysis of data: AJP, RA, PM, RK; drafting of manuscript: AJP, RA, RK; critical revision: AJP, RA, PM, RK, KSD, VK; administrative support: AJP, RA, KSD, VK; and supervision: RA, KSD, VK.

\section{References}

1. Akutagawa T, Tani T, Kida K, et al. A new method for characterizing hand dysfunction in cervical spondylotic myelopathy: a preliminary study. Spinal Cord 2016;54:221-5.

2. Fehlings MG, Tetreault L, Hsieh PC, Traynelis V, Wang MY. Introduction: degenerative cervical myelopathy: diagnostic, assessment, and management strategies, surgical complications, and outcome prediction. Neurosurg Focus 2016;40:E1.

3. Ono K, Ebara S, Fuji T, Yonenobu K, Fujiwara K, Yamashita K. Myelopathy hand: new clinical signs of cervical cord damage. J Bone Joint Surg Br 1987;69:215-9.

4. Yukawa Y, Kato F, Ito K, et al. "Ten second step test" as a new quantifiable parameter of cervical myelopathy. Spine (Phila Pa 1976) 2009;34:82-6.

5. Omori M, Shibuya S, Nakajima T, et al. Hand dexterity impairment in patients with cervical myelopathy: a new quantitative assessment using a natural prehension movement. Behav Neurol 2018;2018:5138234.

6. Doita M, Sakai H, Harada T, et al. Evaluation of impairment of hand function in patients with cervical myelopathy. J Spinal Disord Tech 2006;19:276-80.

7. Hosono N, Takenaka S, Mukai Y, et al. Postoperative 24-hour result of 15-second grip-and-release test correlates with surgical outcome of cervical compression myelopathy. Spine (Phila Pa 1976) 2012;37:1283-7. 
8. Prabhu K, Babu KS, Samuel S, Chacko AG. Rapid opening and closing of the hand as a measure of early neurologic recovery in the upper extremity after surgery for cervical spondylotic myelopathy. Arch Phys Med Rehabil 2005;86:105-8.

9. Shibuya R, Wada E, Iwasaki M, Yonenobu K, Yoshikawa $\mathrm{H}$. Motor conduction measurement in myelopathy hand. Funct Neurol 2014;29:177-82.

10. Doita M, Sakai H, Harada T, et al. The influence of proprioceptive impairment on hand function in patients with cervical myelopathy. Spine (Phila Pa 1976) 2006;31:1580-4.

11. Yoo JS, Ahn J, Mayo BC, et al. Improvements in grip and pinch strength and patient-reported outcomes after anterior cervical discectomy and fusion. Clin Spine Surg 2019;32:403-8.

12. Egwu MO, Ajao BA, Mbada CE, Adeoshun IO. Isometric grip strength and endurance of patients with cervical spondylosis and healthy controls: a comparative study. Hong Kong Physiother J 2009;27:2-6.

13. Liu XY, Yuan SM, Tian YH, Zheng YP, Li JM. Expansive open-door laminoplasty and selective anterior cervical decompression and fusion for treatment of multilevel cervical spondylotic myelopathy. Orthop Surg 2011;3:161-6.

14. Tetreault L, Kopjar B, Nouri A, et al. The modified Japanese Orthopaedic Association scale: establishing criteria for mild, moderate and severe impairment in patients with degenerative cervical myelopathy. Eur Spine J 2017;26:78-84.

15. Mullerpatan RP, Karnik G, John R. Grip and pinch strength: normative data for healthy Indian adults. Hand Ther 2013;18:11-6.

16. Bohannon RW. Muscle strength: clinical and prognostic value of hand-grip dynamometry. Curr Opin Clin Nutr Metab Care 2015;18:465-70.
17. Mendes J, Azevedo A, Amaral TF. Handgrip strength at admission and time to discharge in medical and surgical inpatients. JPEN J Parenter Enteral Nutr 2014;38:481-8.

18. Matos CM, Silva LF, Santana LD, et al. Handgrip strength at baseline and mortality risk in a cohort of women and men on hemodialysis: a 4-year study. J Ren Nutr 2014;24:157-62.

19. Kilgour RD, Vigano A, Trutschnigg B, Lucar E, Borod M, Morais JA. Handgrip strength predicts survival and is associated with markers of clinical and functional outcomes in advanced cancer patients. Support Care Cancer 2013;21:3261-70.

20. Bohannon RW. Hand-grip dynamometry predicts future outcomes in aging adults. J Geriatr Phys Ther 2008;31:3-10.

21. Roumen RM, Hesp WL, Bruggink ED. Unstable Colles' fractures in elderly patients: a randomised trial of external fixation for redisplacement. J Bone Joint Surg Br 1991;73:307-11.

22. Tremayne A, Taylor N, McBurney H, Baskus K. Correlation of impairment and activity limitation after wrist fracture. Physiother Res Int 2002;7:90-9.

23. Kennedy D, Jerosch-Herold C, Hickson M. The reliability of one vs. three trials of pain-free grip strength in subjects with rheumatoid arthritis. J Hand Ther 2010;23:384-90.

24. Bae JH, Kang SH, Seo KM, Kim DK, Shin HI, Shin HE. Relationship between grip and pinch strength and activities of daily living in stroke patients. Ann Rehabil Med 2015;39:752-62.

25. Wang SF, Li PC, Xue YH, Yiu HW, Li YC, Wang HH. Contralateral C7 nerve transfer with direct coaptation to restore lower trunk function after traumatic brachial plexus avulsion. J Bone Joint Surg Am 2013;95:821-7. 